\title{
Corrosion Rate Evaluation on Steel in Concrete Exposed to Sulfate Solutions
}

\author{
M.A. Baltazar, F. Almeraya-Calderón, " V. Orozco Carmona, D. Nieves \\ Mendoza, C. Gaona Tiburcio, A. Borunda Terrazas, A. Martínez Villafañe \\ Centro de Investigación en Materiales Avanzados, S.C. División de Deterioro de Materiales e \\ Integridad Estructural/Grupo Corrosión Miguel de Cervantes 120, Complejo Industrial \\ Chihuahua 31109,Chihuahua, Chih., México
}

Received 26 February 2004; accepted in revised form 24 May 2004

\begin{abstract}
The present work shows the results of the electrochemical evaluation of reinforced concrete specimens exposed in 3 to $5 \% \mathrm{Na}_{2} \mathrm{SO}_{4}$ solutions during four months exhibition. The concrete was elaborated with water/cement ratio $(\mathrm{w} / \mathrm{c})$ of $0.4,0.5$ and 0.6. The electrochemical evaluation was carried out using the lineal resistance polarization technique. The results of these tests show that the biggest attack for $\mathrm{Na}_{2} \mathrm{SO}_{4}$ was presented in the specimens of 0.5 and $0.6 \mathrm{w} / \mathrm{c}$ ratio. Also, they showed a passive tendency after curing. On the other hand the Rp curves analysis, shows that in all the evaluated specimens a great corrosion rate was presented during the curing stage and after some time the system tends to be stabilized approximately in $0.05 \mathrm{~mm} / \mathrm{yr}$.
\end{abstract}

Keywords: concrete, w/c ratio, lineal polarization resistance, corrosion rate.

\section{Introducción}

En la actualidad y desde hace tiempo, el material de construcción más difundido es el hormigón, que junto con las varillas de acero forman el sistema conocido como hormigón armado. Sin embargo, uno de los problemas más importantes para el mantenimiento de la integridad estructural de obras civiles como puentes, túneles, carreteras, muelles, etc., es la corrosión de las varillas de los sistemas de hormigón armado. 
Cuando las condiciones de servicio cambian y el hormigón se altera, o a través de el penetran sustancias agresivas, la corrosión de las varillas se desencadena con una triple consecuencia: a) el acero disminuye su sección o incluso se convierte completamente en óxido; b) debido a las presiones que ejerce el óxido al generarse, el hormigón puede fisurarse o desintegrarse; y c) la adherencia varillahormigón disminuye o desaparece.

Aunque teóricamente son muy numerosos los factores que influyen en la iniciación de la corrosión de varillas, son solo unos pocos los que tienen la capacidad de alterar la pasividad natural de las varillas de refuerzo en el hormigón.

Los iones sulfato son iones despasivantes que intervienen en la degradación del hormigón, lo cual puede permitir que el acero del armado quede expuesto al medio, originándose así su corrosión. El ión sulfato $\left(\mathrm{SO}_{4}{ }^{2-}\right)$ se encuentra principalmente en las aguas de mar, en las aguas residuales industriales en forma de disolución diluida de ácido sulfúrico. En las aguas subterráneas, pocas veces aparece el ión sulfato libre, siendo mucho más frecuentes sus sales, es decir, los sulfatos $[1,7]$.

La presencia de sulfatos en un agua que está en contacto con una pasta endurecida de cemento, puede incrementar considerablemente solubilidad de los componentes de dicha pasta y causar, por una parte el desarrollo de la degradación del concreto por lixiviación. Por otro lado, la presencia de sulfatos puede ocasionar una reacción de cambio de base, en el curso de la cual el catión del sulfato será sustituido por $\mathrm{Ca}^{2+}$, originándose la degradación del concreto, por reacción de cambio iónico. En ciertas circunstancias puede ocurrir, sin embargo, que la presencia de sulfatos dé lugar a una degradación por expansión debido a la formación de otros componentes estables en la masa endurecida del cemento [2]. Una de las causas de la nocividad de los sulfatos es el yeso que se forma al reaccionar el anión sulfato del agua con el $\mathrm{Ca}(\mathrm{OH})_{2}$ liberado por el cemento, el cual se deposita en los poros del hormigón en donde se cristaliza con absorción de dos moléculas de agua. Este proceso de reacción química y cristalización, que es acompañado de un incremento de volumen, ejerce una acción expansiva en los 
poros del hormigón (especialmente en los de las capas superficiales) y termina por lograr su desmoronamiento. Se ha indicado que este aumento de volumen es del 17.7\%. Existen además iones $\mathrm{Mg}^{2+}$, la formación de hidróxido magnésico produce un aumento de volumen del 19.6\%, aproximadamente [3].

Aunado a lo anterior se presenta por el mismo fisuramiento la penetración en la estructuras de los iones sulfatos, y pueden provocar despasivación del acero del armado y generar la formación de pilas electroquímicas, desencadenando la corrosión del acero del armado, pudiendo llegar al colapso de la estructura. Generalmente un hormigón que es atacado por sulfatos tiene una apariencia blanquecina característica. Al ir penetrando poco a poco se va dando una pérdida de rigidez y resistencia del hormigón. Estos efectos se muestran como dos problemas mas frecuentes en la preservación y conservación de las estructuras de hormigón. De ahí la importancia de saber más del comportamiento o la respuesta del hormigón armado a la presencia de agentes agresivos en este caso el sulfato de sodio [4.6], que nos permita tener una idea clara de las acciones a emplear para poder prever daños a la estructura, y con ello cumplir con la durabilidad para la que fue diseñada la estructura.

\section{Desarrollo experimental}

Se fabricaron 9 probetas de hormigón, con una sección cilíndrica de $30 \mathrm{~cm}$ de altura y $15 \mathrm{~cm}$ de diámetro, de acuerdo a las Normas ASTM C470-87 y C192-81 (ver Fig. 1), 3 probetas de relación a/c $=0.4$, de los que uno permaneció inmerso en agua (espécimen patrón), otro en una solución de $\mathrm{Na}_{2} \mathrm{SO}_{4}$ al 3\%, y otro en una solución de $\mathrm{Na}_{2} \mathrm{SO}_{4}$ al $5 \%$. Así mismo para las relaciones a/c 0.5 y 0.6 .

Se empleo la siguiente nomenclatura:

- el primer número por ejemplo 4, 5, 6 se refiere a la relación a/c de la probeta;

- la B se refiere a que es una probeta patrón (inmerso en agua);

- el 3S se refiere a una probeta inmersa en una solución de $\mathrm{Na}_{2} \mathrm{SO}_{4}$ al 3\%;

- el $5 \mathrm{~S}$ se refiere a una probeta inmersa en una solución de $\mathrm{Na}_{2} \mathrm{SO}_{4}$ al 5\%.

En cada cilindro se embebieron tres varillas de acero al carbono, haciendo cada una de ellas la función de electrodo de trabajo, electrodo auxiliar y electrodo de 
referencia. A todas las varillas se les colocó una franja de $3 \mathrm{~cm}$ de cinta de fluorocarbono cubriendo desde los $27 \mathrm{~cm}$ a los $30 \mathrm{~cm}$; la finalidad de cubrir las varillas con cinta de fluorocarbono, es evitar que se presenten zonas de aeración diferencial, concentración de sales o en algunos casos que esta zona se comporte como hendidura, presentándose de esta manera corrosión por hendidura.

Posteriormente se llevó a cabo una etapa de curado de 28 días después del fraguado, mismo tiempo que las probetas estuvieron inmersas en agua para cumplir con las recomendaciones [5]. Después del tiempo de curado las probetas consideradas patrones ( 3 probetas), se quedaron en la tina de curado; las restantes 6 probetas se expusieron en soluciones de $\mathrm{Na}_{2} \mathrm{SO}_{4}$ al 3 y $5 \%$.

Para la evaluación electroquímica se empleo la técnica de resistencia a la polarización ( $\mathrm{Rp}$ ), con un barrido $\pm 20 \mathrm{mV}$ vs. $\mathrm{E}_{\text {corr }} \mathrm{y}$ una velocidad de barrido de $10 \mathrm{mV} / \mathrm{min}$. Las pruebas se realizaron una vez por semana durante 15 semanas, de las cuales, las 4 primeras semanas fueron en la etapa curado, las siguientes fueron las de exposición en $\mathrm{Na}_{2} \mathrm{SO}_{4}$.

\section{Resultados}

A continuación se presenta un condensado de las curvas obtenidas mediante la técnica electroquímica de resistencia a la polarización. Cabe señalar que las primeras 4 semanas de exposición fueron correspondientes a la etapa de curado.

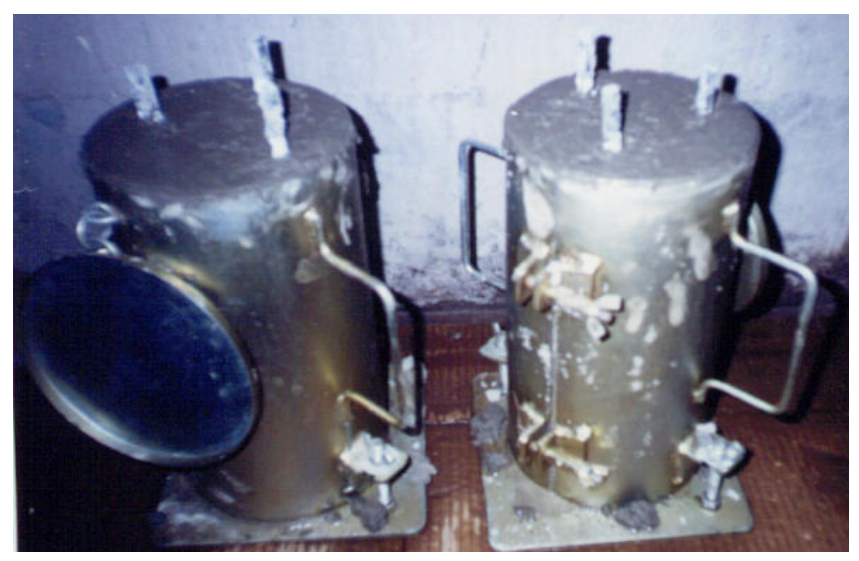

Figura 1. Probeta fabricada de hormigón. 
En las gráficas 1, 2 y 3 se observa un comportamiento similar en los tres medios de exposición (agua, $\mathrm{Na}_{2} \mathrm{SO}_{4}$ al 3\% y 5\%) siendo, inicialmente (etapa de curado) un estado activo evidenciándose en mayor grado en las probetas con relación a/c de 0.5 y 0.6 , presentando una mayor resistencia a la corrosión la probeta con relación a/c de 0.4; posterior a esta etapa de curado (a partir de la 5a. semana de exposición) todas las probetas presentaron una disminución considerable en la densidad de corriente de corrosión, por lo tanto, un aumento en la resistencia a la polarización. Acentuándose mayormente en la probeta con relación a/c de 0.4, atribuyéndose lo anterior a que en una relación a/c de 0.4 la cantidad de poros en la masa del concreto es mínima.

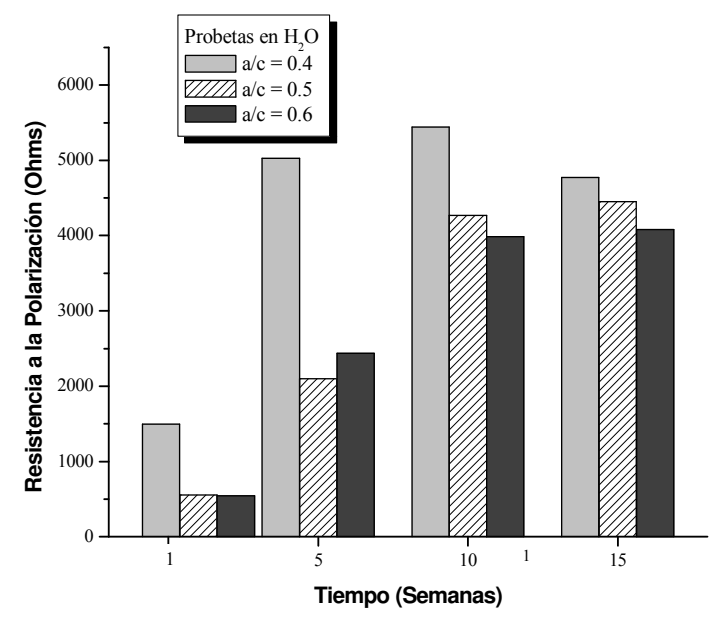

Gráfica 1. Rp para las probetas con relación a/c $0.4,0.5$ y 0.6 en agua.

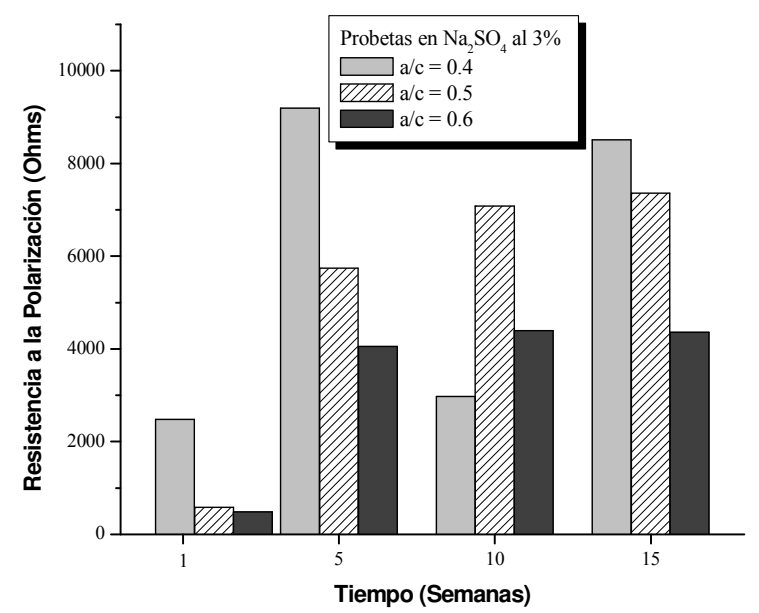

Gráfica 2. Rp para las probetas con relación a/c $0.4,0.5$ y 0.6 en $\mathrm{Na}_{2} \mathrm{SO}_{4}$ al 3\%. 


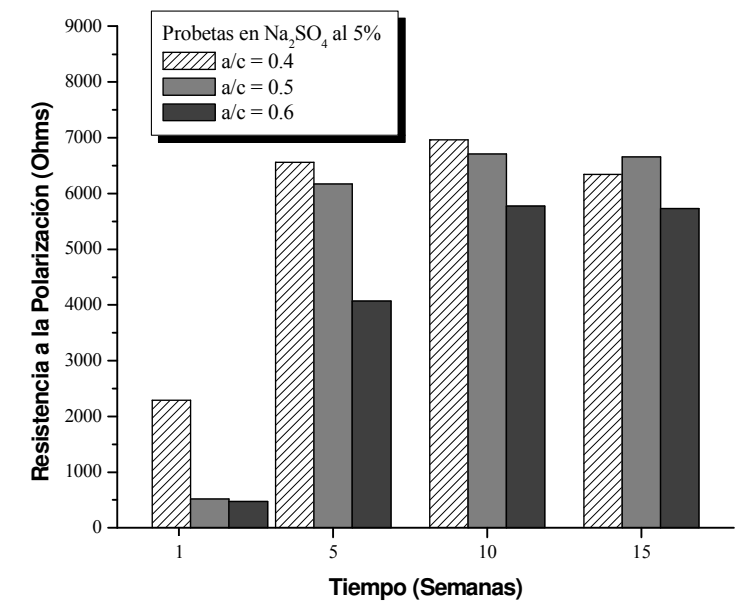

Gráfica 3. Rp para las probetas con relación a/c de $0.4,0.5$ y 0.6 en $\mathrm{Na}_{2} \mathrm{SO}_{4}$ al $5 \%$.

En las gráficas de velocidades de corrosión en función de las semanas de exposición, se analizaron las probetas contra diferentes relaciones a/c y los medios en que estuvieron expuestos.

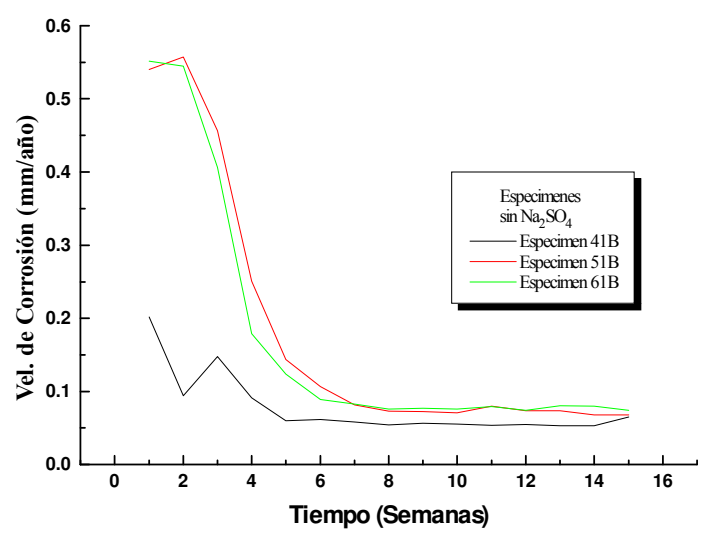

Gráfica 4. Comportamiento de la velocidad de corrosión en probetas de diferente relación $\mathrm{a} / \mathrm{c}$, expuestos únicamente en agua.

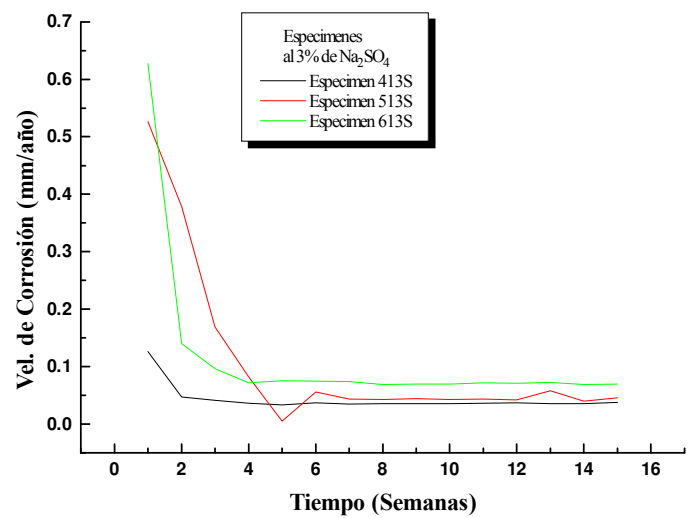

Gráfica 5. Comportamiento de la velocidad de corrosión en probetas de diferente relación a/c, expuestos en una solución de $\mathrm{Na}_{2} \mathrm{SO}_{4}$ al 3\%. 


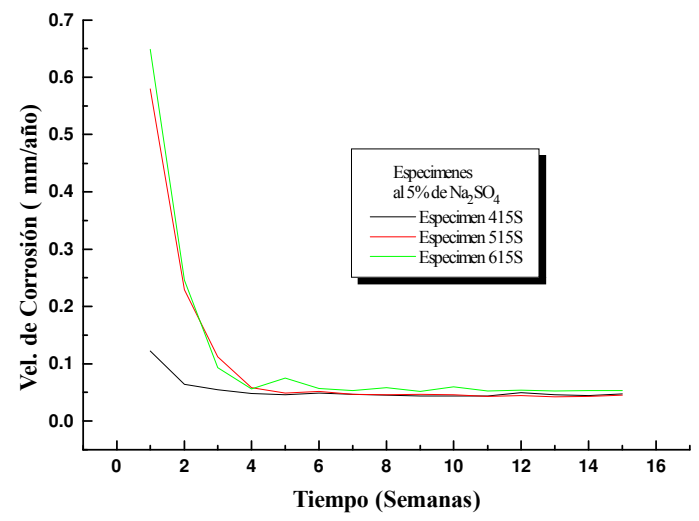

Gráfica 6. Comportamiento de la velocidad de corrosión en probetas de diferente relación a/c, expuestos en una solución de $\mathrm{Na}_{2} \mathrm{SO}_{4}$ al 5\%.

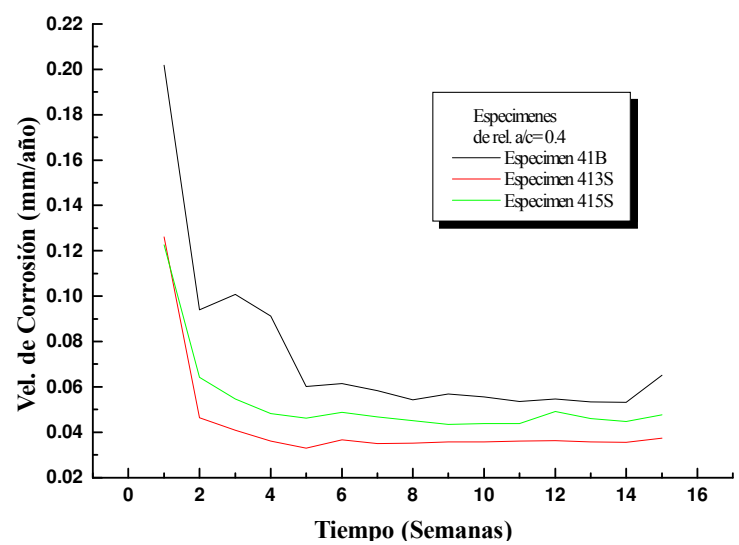

Gráfica 7. Comportamiento de la velocidad de corrosión en probetas con relación a/c de 0.4 , expuestos en los tres medios de experimentación.

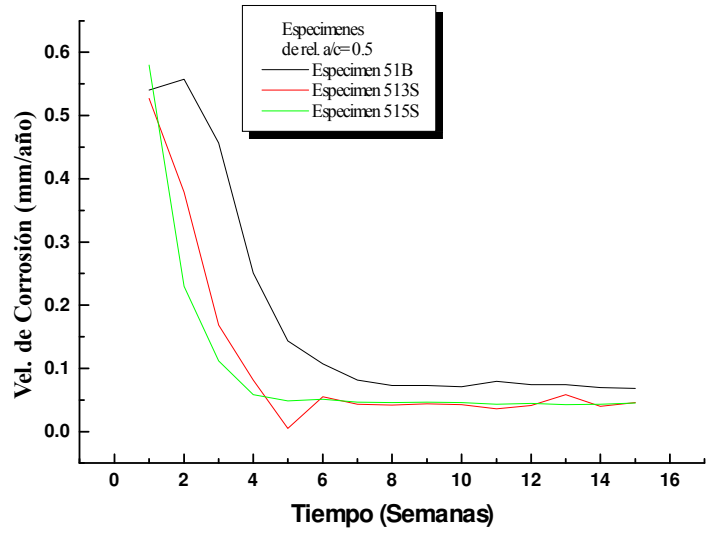

Gráfica 8. Comportamiento de la velocidad de corrosión en probetas con relación a/c de 0.5 , expuestos en los tres medios de experimentación

Se hace notar que en general (gráficas 4, 5 y 6) se observó una disminución en la velocidad de corrosión en las tres probetas a medida que se incrementa el tiempo 
de exposición, hasta estabilizarse en una vel $_{\text {corr }}$ de $0.05 \mathrm{~mm} /$ año aproximadamente, además se visualizó una menor velocidad en la probeta con una relación a/c de 0.4 en los tres medios de exposición, siendo el comportamiento similar en las probetas de relación a/c de 0.5 y 0.6 .

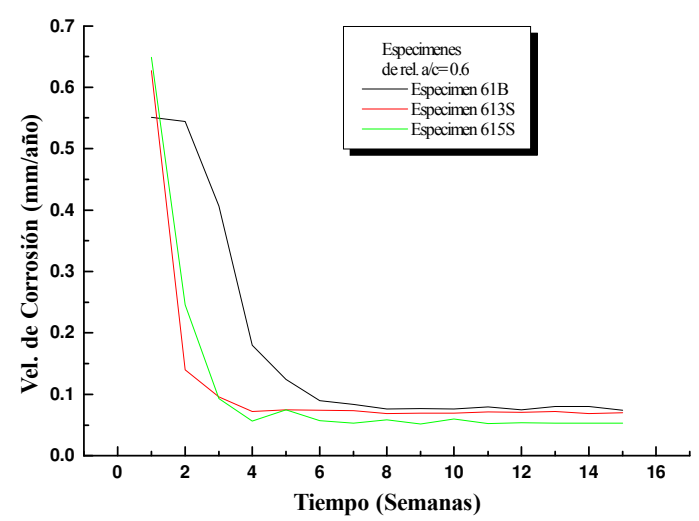

Gráfica 9. Comportamiento de la velocidad de corrosión en probetas con relación a/c 0.6, expuestos en los tres medios de experimentación

Al analizar las gráficas 7, 8 y 9 que compara las velocidades de corrosión de las probetas con una misma relación a/c, en presencia de agua, $\mathrm{Na}_{2} \mathrm{SO}_{4}$ al $3 \%$ y $5 \%$, se

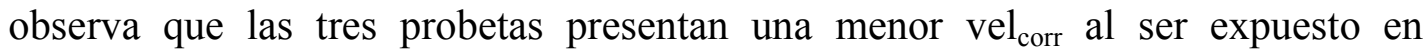
soluciones de $\mathrm{Na}_{2} \mathrm{SO}_{4}$ al 3 y $5 \%$. En las tres gráficas también se puede observar que conforme se aumenta el tiempo de exposición la vel $\mathrm{c}_{\text {corr }}$ tiende a disminuir y ser prácticamente constante.

\section{Conclusiones}

- El comportamiento de la $\mathrm{Vel}_{\text {corr }}$ presente en las varillas del armado en todas las probetas en evaluación fue similar, iniciando con un valor relativamente elevado (durante la etapa de curado), disminuyendo conforme aumenta el tiempo de exposición, llegando a establecerse en un valor que se puede considerar constante (aproximadamente $0.05 \mathrm{~mm} / \mathrm{año}$ ).

- Se observó durante la evaluación de las probetas en los tres diferentes medios de exposición (agua, $\mathrm{Na}_{2} \mathrm{SO}_{4}$ al 3 y 5\%), que las probetas con relación a/c de 0.4 presentaron una mayor resistencia la corrosión que las probetas con las relaciones a/c de 0.5 y 0.6 , atribuyéndose lo anterior a que en una relación a/c de 0.4 la cantidad de poros en la masa del hormigón se reduce. 
- Se encontró que todas las probetas (relaciones a/c de $0.4,0.5$ y 0.6 ) inmersos en agua $\sin \mathrm{Na}_{2} \mathrm{SO}_{4}$, presentaron velocidad de corrosión más alta que cuando fueron expuestos en soluciones de $\mathrm{Na}_{2} \mathrm{SO}_{4}$ al 3 y $5 \%$.

\section{Evaluación de la Velocidad de Corrosión del Acero en Hormigón Expuesto a Soluciones de Sulfatos}

\section{Resumen}

En el presente trabajo se muestran los resultados de la evaluación electroquímica de probetas de hormigón armado expuestos en soluciones de $\mathrm{Na}_{2} \mathrm{SO}_{4}$ al 3 y $5 \%$, durante cuatro meses de exposición. El hormigón fue elaborado con una relación agua/cemento $(\mathrm{a} / \mathrm{c})$ de $0.4,0.5$ y 0.6 . La evaluación electroquímica se realizó empleando la técnica de resistencia a la polarización lineal. Los resultados de estas probetas muestran que el mayor ataque por $\mathrm{Na}_{2} \mathrm{SO}_{4}$ se presenta en los especimenes con una relación a/c de $0.5 \mathrm{y}$ 0.6 , además, mostraron una tendencia pasivante posterior a la etapa de curado. Por otro lado el análisis de la curvas de $\mathrm{Rp}$, mostraron en todas las probetas evaluadas, una mayor velocidad de corrosión en la etapa de curado, la cual, conforme se avanzo en el estudio tendió a estabilizarse en un valor aproximadamente de $0.05 \mathrm{~mm} / \mathrm{año}$.

Palabras claves: hormigón, relación a/c, resistencia a la polarización lineal, velocidad de corrosión.

\section{Referencias}

1. Manual de Inspección, Evaluación y Diagnóstico de Corrosión en Estructuras de Hormigón Armado. Durar, Red temática del CYTED, 1997.

2. D. Dal Molin, "Fissuras em Estructuras de Concreto Armado: Análise das Manifestações Típicas e Levantamento de Casos Ocurridos no Estado do Rio Grande do Sul”. Dissertação (Maestrado). Curso de Pós Graduação em Engenharia Civil, Universidade Federal do Rio Grande do Sul. Porto Alegre, Brasil,1988.

3. G.C. Mays, "The Behaviour of Concrete". Durability of Concrete Structures. Investigation, Repair, Protection. Edited by Geoff Mays. U.K. 1992.

4. A. Bretaña, D. Dal Molin, "Resistencia de cemento frente al ataque por sulfatos", V Congreso Iberoamericano de Patologías de la Construcción. VII Congreso de Control de Calidad. Vol. 1. Pags. 295-289. 
5. L.O. Herrera, "Corrosión del concreto reforzado en pruebas aceleradas", U.A.CH. 1998.

6. M.C. Andrade, C. Alonso, Construction and Building Materials 10 (1996) 315-328.

7. Tiewei Zhang, Odd E. Gjorv, Cement and Concrete Research 24 (1994) 1534-1548. 